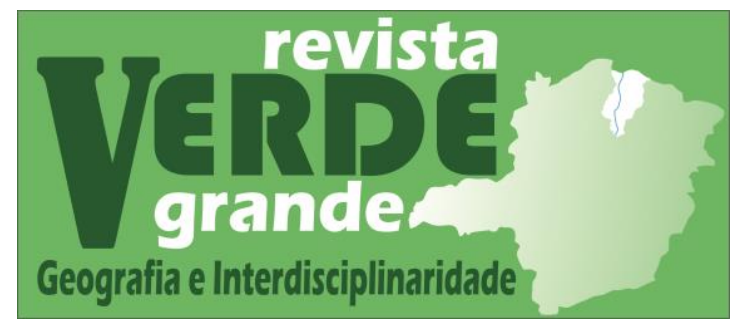

\title{
HIDROPAISAGENS DO VALE DO AÇU: A EXPERIÊNCIA DE UM PROJETO DE ENSINO
}

\author{
Hydrolandscapes of the Açu valley in: the experience of a teaching project
}

\author{
Josiel de Alencar Guedes ${ }^{1}$ https://orcid.org/0000-0001-6436-563X. \\ Gerônimo da Silva Costa ${ }^{2}$ https://orcid.org/0000-0003-2695-9263 \\ Aluízio Bezerra Júnior ${ }^{3}$ https://orcid.org/0000-0003-4455-6935
}

\footnotetext{
1 Prof. Dr. Curso de Geografia - Universidade do Estado Rio Grande do Norte (UERN), Campus de Açú. Email: josielguedes@uern.br.

2 Graduado em Geografia - Universidade do Estado do Rio Grande do Norte (UERN), Campus de Acú. Email: geronimosilvacosta@hotmail.com.

${ }^{3}$ Graduado em Geografia e Mestrando no Programa de Pós-Graduação em Geografia (PPGEO) - Universidade do Estado do Rio Grande do Norte (UERN), Campus Central. Email: aluiziojunior@ outlook.com
}

\section{Resumo}

Este relato de experiência visa apresentar um projeto de ensino associado à disciplina Hidrografia, ministrada no semestre 2019.1 e ofertada para o $4^{\circ}$ período do curso de Geografia da Universidade do Estado do Rio Grande do Norte (Campus de Açú). O projeto teve como objetivo mapear reservatórios e lagoas no Vale do Açu (RN) utilizando imagens do Google Earth o programa de geoprocessamento QGIS. Nas aulas, foram apresentadas as imagens dos mananciais superficiais e discutidos problemas relacionados a cada um deles. Em seguida, foram realizadas as aulas em campo, onde os alunos fizeram registros de problemas específicos e se discutiu a relação de uso do solo no seu entorno. Posteriormente, foram elaboraram os mapas de cada manancial. A experiência do projeto mostrou-se importante ao se estudar a disciplina com exemplos de mananciais mais perto da realidade dos alunos.

Palavras-chave: Hidrografia. Geoprocessamento. Mapas temáticos.

\begin{abstract}
This experience report aims to present a teaching project associated with the discipline Hydrography, taught in the semester 2019.1 and offered for the 4th period of the Geography course of the State University of Rio Grande do Norte (Campus de Açú). The project aimed to map reservoirs and lagoons in the Açu Valley (RN) using Google Earth images of the QGIS geoprocessing program. In the classes, images of the surface springs were presented and problems related to each one were discussed. Then, the classes were held in the field, where the students made records of specific problems and the relationship of land use in their surroundings was discussed. Subsequently, the maps of each spring were elaborated. The experience of the project proved to be important when studying the discipline with examples of springs closer to the reality of the students.
\end{abstract}

Keywords: Hydrography. Geoprocessing. Themathic maps. 


\section{A hidropaisagem e o projeto de ensino}

A Universidade do Estado do Rio Grande do Norte, em resolução própria (UERN, 2017, p.1), regulamenta a oferta de projetos de ensino, “[...] caracterizando-o como um conjunto de atividades acadêmicas vinculadas aos cursos de graduação que visa aprimorar a qualidade do processo de ensino-aprendizagem docente e discente da UERN". A partir desse entendimento, foi pensado uma proposta de projeto que associasse uma prática a uma disciplina.

O projeto de ensino foi desenvolvido na disciplina "Hidrografia", ofertada no $4^{\circ}$ período do curso de Geografia do Campus de Açu. Durante a apresentação do Programa Geral do Componente Curricular (PGCC) foi informado à turma que haveria o desenvolvimento dessa atividade e que constaria com a participação de dois alunos sendo um deles, à época, monitor da disciplina e o outro aluno do Programa de Pós-graduação em Geografia (PPGEO/UERN) em estágio de docência.

As atividades foram desenvolvidas em quatros etapas: a primeira constou de um levantamento de bibliografia sobre hidrossistemas e depois associados à discussão da disciplina relacionados aos mananciais do superficiais do Vale; na segunda etapa foram selecionadas imagens do Google Earth que mostrassem os mananciais existentes no Vale do Açu, sendo também incorporadas à discussão da disciplina; na terceira etapa houve o treinamento no Laboratório de Informática, auxiliado pelos alunos colaboradores, da ferramenta de geoprocessamento QGIS, com versões abertas disponíveis na internet (QGIS, 2019), onde foram elaborados os mapeamentos temáticos dos mananciais. Em campo, quarta etapa, foram explanadas informações sobre os respectivos mananciais, coletados dados primários e registros fotográficos.

A turma foi dividida em quatro grupos com a supervisão do professor orientador e dos alunos colaboradores, ficando a cargo deles a elaboração dos mapeamentos temáticos dos mananciais a partir de imagens do Google Earth.

Foram eleitos para a pesquisa, discussão em campo e mapeamento de mananciais, a lagoa de Piató (Figura 1), considerada uma das maiores mais importantes na região, um complexo de lagoas temporárias (Figura 2) que se interligam entre si quando do período chuvoso na região, e os reservatórios Mendubim(Figura 3) e o de Itajá (Figura 4). 


\section{Hidropaisagens do Vale do Açu: a experiência de um projeto de ensino}

Josiel de Alencar Guedes; Gerônimo da Silva Costa; Aluízio Bezerra Júnior

Figura 1: Lagoa de Piató (Assú RN)

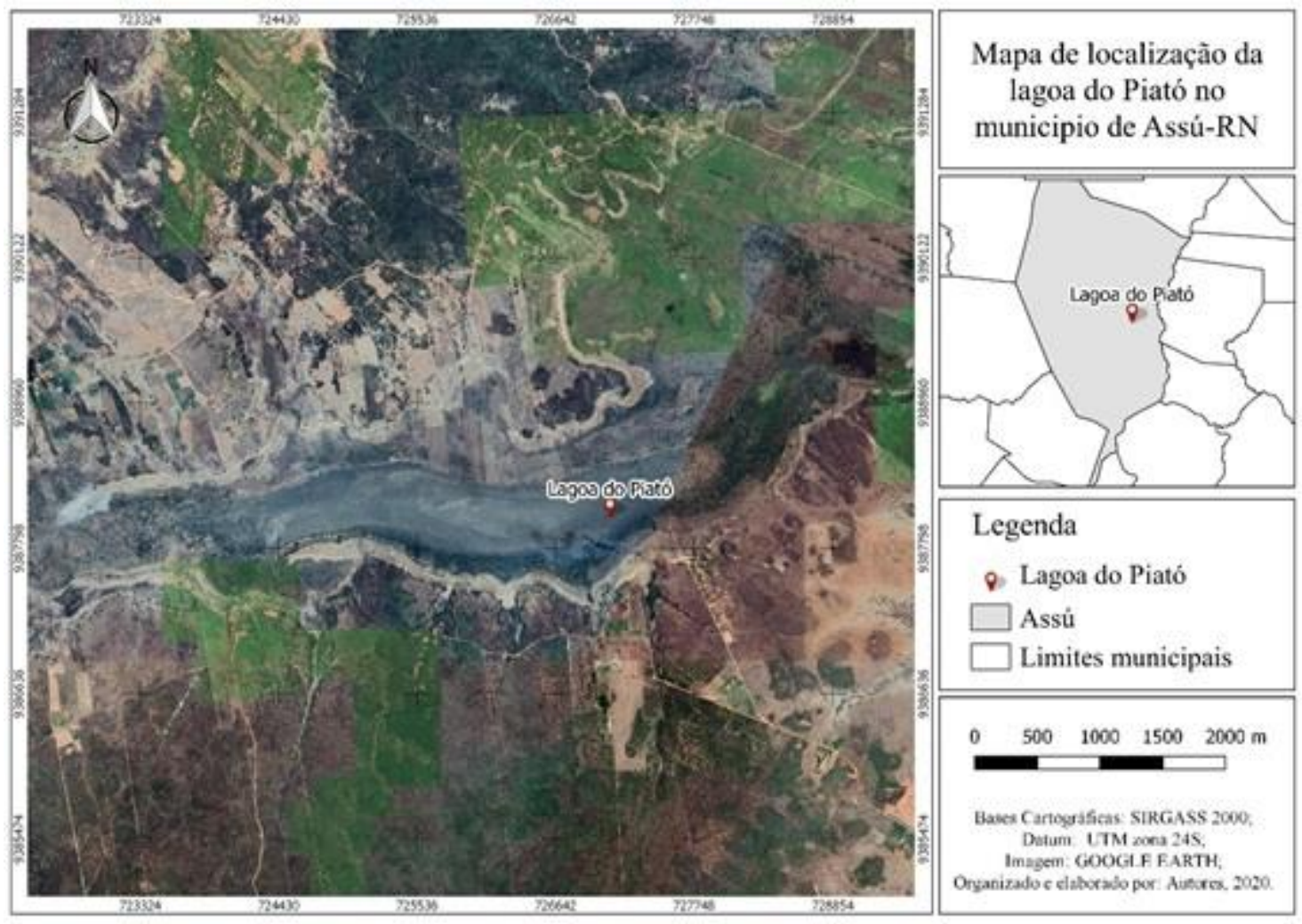

Fonte: Imagens obtidas no Google Earth

Figura 2: Complexo de lagoas (Assú, RN)

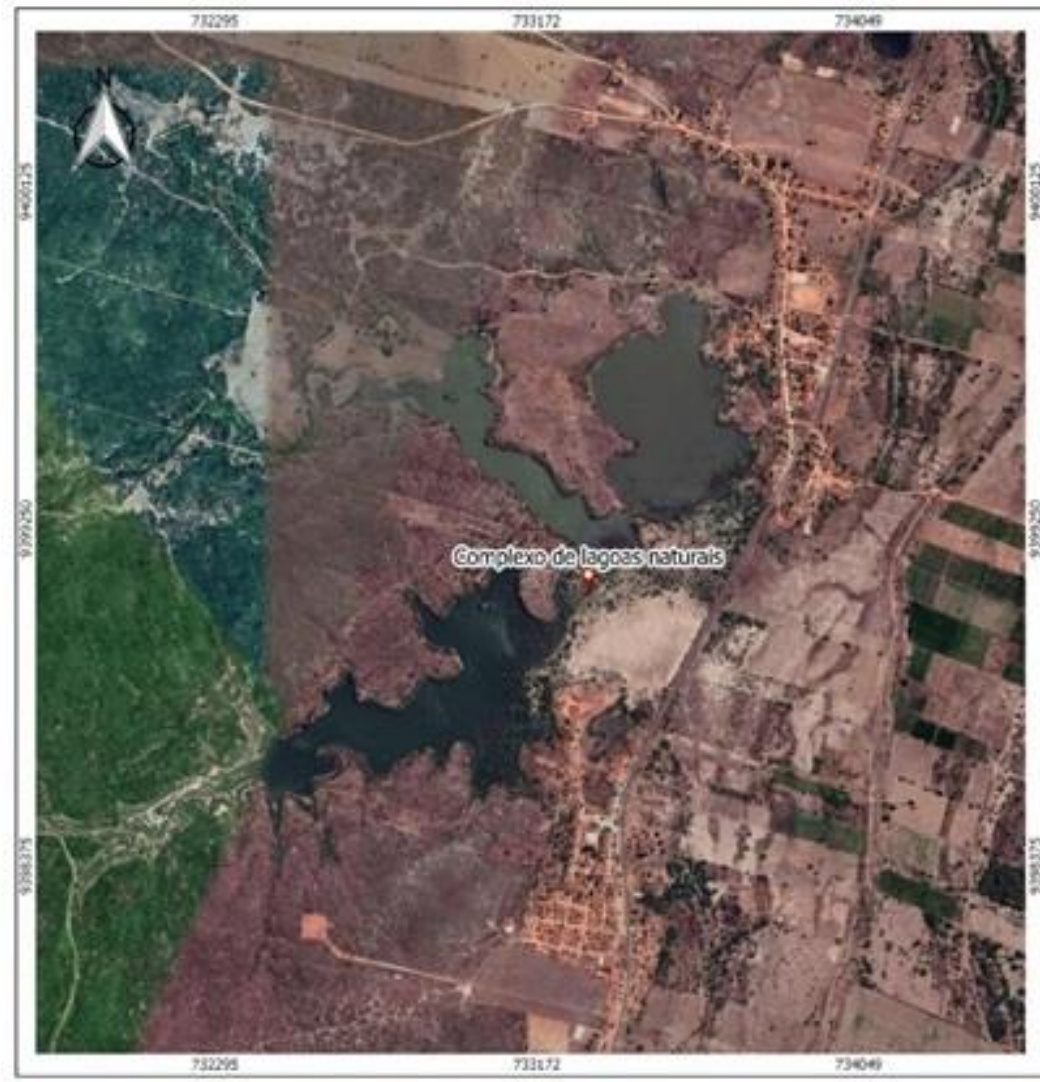

Mapa de localização do complexo de lagoas naturais no municipio de Assú-RN

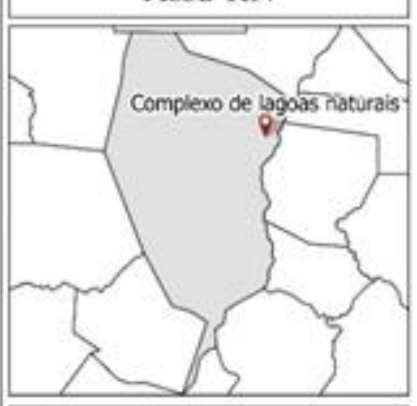

Legenda

Q Complexo de lagoas

$\square$ Assú

$\square$ Limites municipais

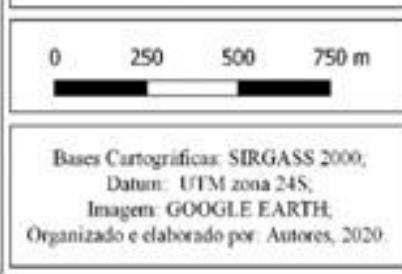

Fonte: Imagens obtidas no Google Earth 


\section{Hidropaisagens do Vale do Açu: a experiência de um projeto de ensino}

Josiel de Alencar Guedes; Gerônimo da Silva Costa; Aluízio Bezerra Júnior

Figura 3: Reservatório de Mendubim (Assú, RN)

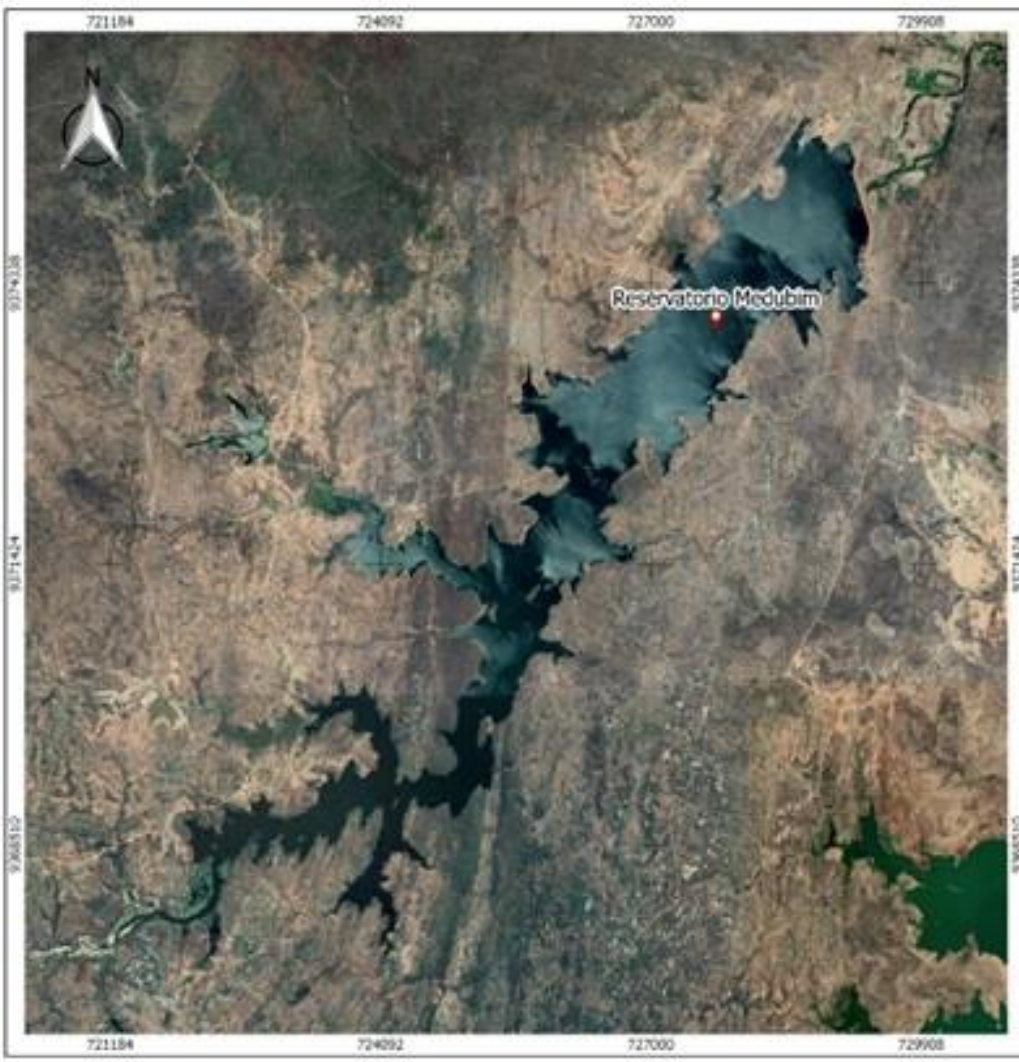

Mapa de localização do reservatorio Medubim no municipio de Assú-RN

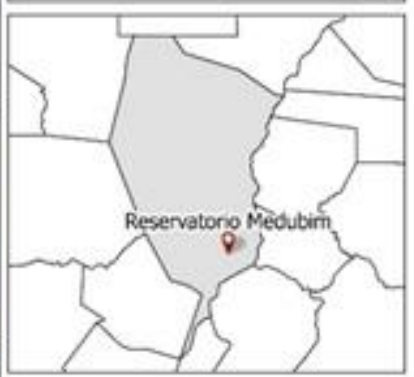

Legenda

Q Reservatório

Assú

Limites municipais

Fonte: Imagens obtidas no Google Earth

Figura 4: Reservatório de Itajá (Itajá, RN)

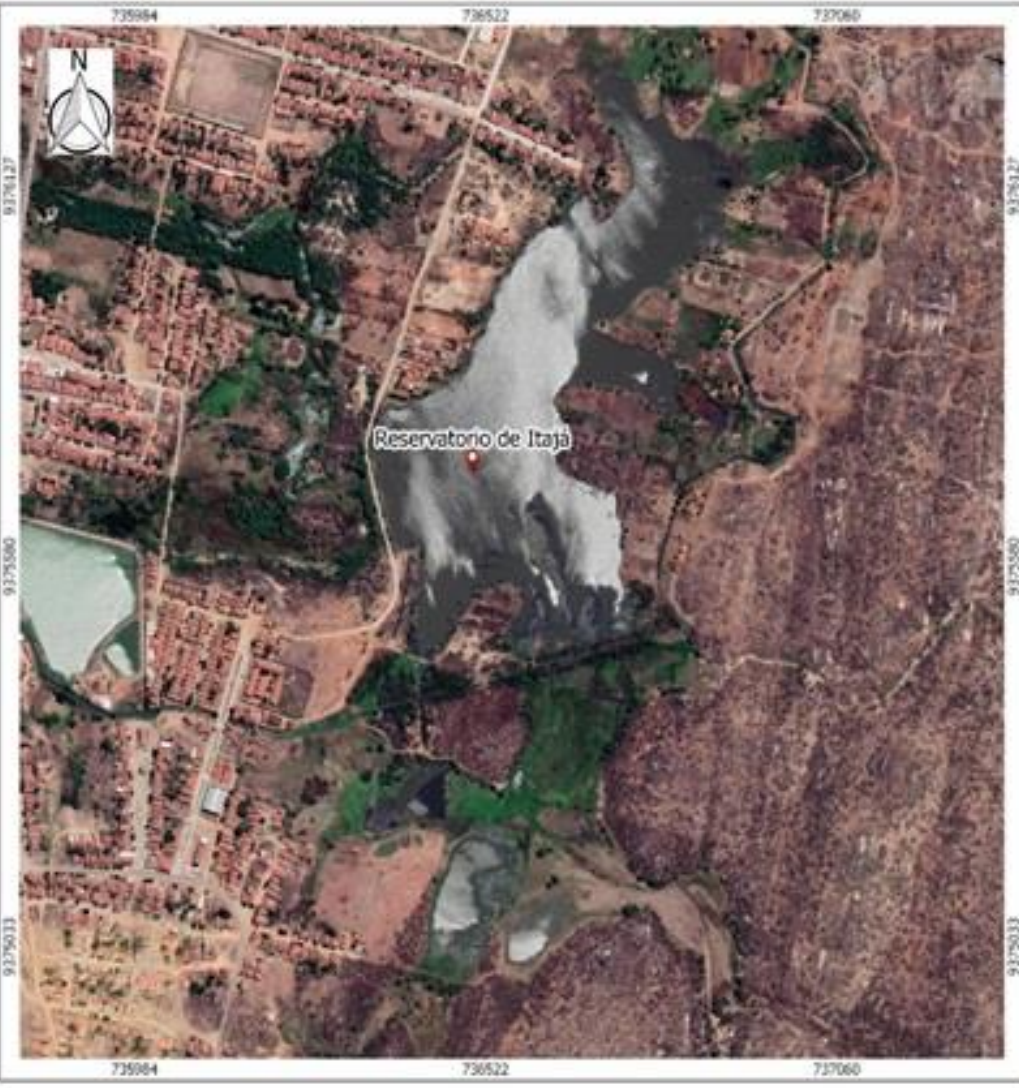

Mapa de localização do reservatório público do municipio de Itajá-RN.

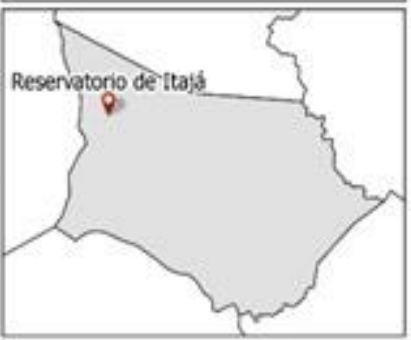

Legenda

Q. Reservatório

$\square$ Itajá

Limites municipais

$0 \quad 100 \quad 200 \quad 300 \mathrm{~m}$

Bases Cartograficas SIRG GSS 2000 Dahin: UTM zona 24S

Imagen: GOOGLEE.ARTI Onganizado e daborado por. Autures, 2020

Fonte: Imagens obtidas no Google Earth 


\section{Hidropaisagens do Vale do Açu: a experiência de um projeto de ensino}

Josiel de Alencar Guedes; Gerônimo da Silva Costa; Aluízio Bezerra Júnior

O trabalho com a ferramenta de geoprocessamento e a ida ao campo possibilitou, aos alunos, novos conhecimentos além de dirimir a lacuna sobre o desconhecimento da realidade local acerca dos sistemas hídricos na região.

As imagens do Google Earth, também disponíveis gratuitamente, serviram bem aos propósitos do projeto. O uso das geotecnologias, associadas às novas metodologias de ensino, podem contribuir efetivamente para um melhor aprendizado, especialmente relacionada à Geografia Física, no caso, o ramo específico a Hidrografia (SANTOS, 2019, SEGALA, 2017, MENEGHOSO, 2014).

O QGIS é um bom programa de geoprocessamento que, além de acesso grátis, é satisfatório para a elaboração dos mapas a serem trabalhados nas disciplinas da graduação e pós-graduação. Houve, no entanto, alguma dificuldade por parte dos alunos em manuseá-lo, em parte associada aos computadores já considerados obsoletos e com pouca memória causando lentidão e, também, relacionada ao suporte da internet. No entanto, fez-se uma recomendação sobre a necessidade de laboratórios específicos com máquinas modernas e com suporte a geotecnologia a ser trabalhada.

O projeto desenvolvido mostrou que é possível associar novas informações práticas e teóricas, especialmente relacionando as disciplinas ofertadas no curso de Geografia à realidade local, uma vez que grande parte dos alunos matriculados não conheciam os mananciais estudados.

\section{Referências}

MENEGHESSO, V.A. A Hidrografia local e as práticas escolares de professores de Geografia de Ibitinga - SP. Dissertação (Mestrado em Educação). Universidade de São Paulo, Faculdade de Filosofia e Letras de Ribeirão Preto, Ribeirão Preto, 2014.

QGIS - Um sistema de Informação Geográfica livre e aberto. Disponível em: <https://www.qgis.org/pt_BR/site/>. Acesso em: nov. 2019.

SANTOS, B. B.; BASTOS, M. N. P.; SILVA, M. M.; VARGAS, K. B. Propostas para o ensino de Hidrogeografia: o lugar como categoria de análise geográfica e o uso de recursos didáticos visuais. Geografia Física e as mudanças globais. Fortaleza: UFC, 2019, p.1-5.

SEGALA, F. J. O processo de ensino de Geografia a partir da hidrografia de Francisco Beltrão, PR. Dissertação (Mestrado em Geografia). Universidade Estadual do Oeste do Paraná.Centro de Ciências Humanas, Francisco Beltrão, 2017.

UERN - Universidade do Estado do Rio Grande do Norte. RESOLUÇÃO No 33/2017 CONSEPE: Regulamenta o Projeto de Ensino de Graduação noscursos de graduação da Universidade do Estado do Rio Grande do Norte. Mossoró, 2017. 